\title{
Regional distribution of steric and mass contributions to sea level changes
}

\author{
Marta Marcos (1), Francesc M. Calafat (1), William Llovel (2), Benoit Meyssignac (2), Damià Gomis (1), and \\ Anny Cazenave (2) \\ (1) IMEDEA(CSIC-UIB), Esporles, Spain (marta.marcos@uib.es), (2) LEGOS, OMP, Toulouse, France
}

\begin{abstract}
At inter-annual and longer time scales regional sea level changes are caused by steric (density) changes and mass addition/subtraction. In turn, the mass component can be separated in a global component (increase/decrease of the mass of the global ocean) and a regional component (mass displacements derived from changes in the atmospheric pressure and wind that average to zero at global scale). Separating the sources and reducing the uncertainties of each contribution to regional sea level rise rates is of key importance to understand the causes of sea level variations and to infer eventual future changes. In this work we address the quantification of these contributions in different regions of the world and examine their consistency with observed sea level variability. To do this we base on altimetric sea level observations, hydrographic data and the independent measures of the mass contribution to sea level changes provided by the GRACE mission since 2002. The comparisons demonstrate that the sea level budget can be closed in most regions around the globe (e.g. in the Atlantic and Pacific Oceans), with the exception of the Indian Ocean. Whether this closure will hold for smaller regions will depend on how good is the coverage of the hydrographic observations and how accurate are the estimated ocean mass variations from GRACE. An uncertainty assessment is thus of primary importance. Assuming that the consistency observed for the period 2002-08 holds for the last decades, we estimate rates of change of the mass component for the period 1950-2003 using total sea level data from a reconstructed field and historical hydrographic observations. A question that remains open is whether the spatial patterns of the mass increase obtained for the last decades can provide information on the sources of the added fresh water.
\end{abstract}

